\title{
ORIGINAL
}

\section{ANÁLISIS DEL EFECTO DE COHORTE EN LA EVOLUCIÓN DE LA MORTALIDAD POR ENFERMEDAD DE LA MOTONEURONA EN ESPAÑA, 1951-1992}

\author{
Javicr Llorea Díaz, M. Dolores Prieto Salceda y Miguel Delgado-Rodríguez
}

Cátedra de Medicina Preventiva y Salud Pública. Facultad de Medicina. Universidad de Cantabria

\section{RESUMEN}

Fundamento: Se ha descrito un aumento en la mortalidad por enfermedad de la motoneurona (EMN) en España en las últimas décadas; se ha sugcrido que cste aumento se debe a un efecto de cohorte, pero esta hipótesis no ha sido comprobada.

Métodos: Los datos de mortalidad por edad y sexo debido a la EMN se han obtenido del Instituto Nacional de Estadística. Se han calculado las tasas de mortalidad específicas por edad y las tasas ajustadas por edad. Mediante regresión de Poisson se ha analizado el efecto de la cohorte de nacimiento en la mortalidad por edades; el efecto de cohorte fue también analizado por métodos gráficos.

Resultados: La mortalidad ajustada por edad descendió hasta 1969 y aumentó desde entonces. Cada cohorte quinquenal de nacimiento aumenta en un 8,5\% el riesgo de morir por EMN.

Conclusiones: El aumento de la mortalidad por EMN en España está causado por un efecto de cohorte.

Palabras clave: Enfermedad de la neurona motora. Mortalidad. Efecto de cohorte.
ABSTRACT

The Analysis of the Cohort Effect on the Trend in the Mortality Due to Motor Neuron Disease in Spain, 1951-1992

Background: An increase in the mortality due to motor neuron disease (MND) has been reported in Spain over the past $30-40$ years. It has been suggested that this increase is due to the cohort effect, but his hypothesis has not been proven.

Methods: The motor neuron disease (MND) mortality statistics by age and by gender were furnished from the Spanish National Institute of Statistics. The mortality specifically by age and the adjusted age-related rates were calculated. By Poisson regression, the cohort effect of birth on the mortality by ages has been analyzed, the cohort effect also having been analyzed by graphic methods.

Results: The mortality adjusted by age was declining up until 1969 , as of which time it has been on the rise. Each five-year birth cohort increases the risk of dying from MND by $8.5 \%$.)

Conclusions: The increase in the mortality due to MND in Spain is the result of a cohort effect.

Key words: Motor neuron disease. Mortality. Cohort effect.

\section{INTRODUCCIÓN}

Diferentes estudios han mostrado una tendencia al aumento en la incidencia y la mortalidad por la enfermedad de la motoneurona $(E M N)$ en varios paises, como Francia ${ }^{1,2}$, Italia $^{3,4}$, Noruega ${ }^{6}$, Suecia ${ }^{7}$, Inglaterra y Gales ${ }^{8}$ y

\footnotetext{
Correspondencia:

Javier Llorca

Departamento de Medicina Preventiva y Salud Pública

Facultad de Medicina

Universidad de Cantabria

Avda. Cardenal Herrera Oria s/n

39011 Santander

Correo electrónico: llorcaj@medi.unican.es
}

Estados Unidos ${ }^{9,10}$. Algunos de estos trabajos sugieren que el aumento no se debe a un efecto de cohorte $\mathrm{y}$ consideran la posibilidad de que se esté produciendo por el aumento en la esperanza de vida, como consecuencia de cambios en los riesgos competitivos de mortalidad, que permiten que la población susceptible consiga sobrevivir hasta las edades en que aparece la $\mathrm{EMN}^{13,6,9}$. Sin embargo, en ninguno de los estudios señalados se realizó un análisis formal del efecto de cohorte.

La evolución de la EMN en España ha sido estudiada por Veiga-Cabo et al, quienes encuentran cifras de mortalidad intermedias 
a nivel internacional $(1,49$ por 100.000$)$, con un descenso en la mortalidad ajustada por edades durante los años 60 y un aumento desde 1970; este comportamiento es común a ambos géneros aunque con una razón varón:mujer de 1,6:1, en promedio. Además muestran un patrón geográfico con mayor mortalidad en el norte ${ }^{11}$. El interesante trabajo de Veiga-Cabo et al —pionero en la evolución de la EMN en España- se realizó por porcedimientos transversales por lo que no se pudo analizar si la evolución encontrada era causada por un efecto de período o de cohorte, aunque los autores sugirieron la hipótesis de un aumento de riesgo en las cohortes nacidas hacia 1930. Un trabajo anterior sobre la EMN en Cantabria, encontró una incidencia de 1,01 por 100.000 habitantes y una prevalencia de 3,52 por 100.000 ; sin embargo, la tendencia en la presentación de la enfermedad no fue analizada ${ }^{12}$.

El objetivo de este trabajo es analizar el efecto de la cohorte y del período sobre el aumento de la mortalidad por EMN en España. Para ello se realizará un análisis de cohorte por métodos gráficos y un análisis de edad y cohorte por regresión de Poisson.

\section{MÉTODOS}

Se obtuvo del Instituto Nacional de Estadística el número de muertes para cada sexo y para cada grupo de edad de 35 años en adelante por EMN en España, desde 1951 hasta 1992. Los datos corresponden a los códigos 356 de la CIE-6 (años 1951 a 1960) y CIE-7 (1961-1967), 348 de la CIE-8 (1968-1979) y 335.2 de la CIE-9 (1980-1992). Estos códigos no hacen distinción entre las diferentes enfermedades de la motoneurona (esclerosis lateral amiotrófica, parálisis bulbar progresiva,...). En las sucesivas ediciones del Movimiento Natural de la Población Española figuran sólo los datos sobre total de muertes, sin separación por edad y sexo; por ello fue necesario solicitar los datos directamente a la sede central del INE. Los datos obtenidos están en grupos decenales desde 1951 hasta
1960, en grupos quinquenales (último grupo 70 años y más) desde 1961 hasta 1970 y en grupos quinquenales (último grupo 85 años y más) desde 1971 en adelante. Se obtuvo la población española en cada año, para cada grupo de edad y sexo, por interpolación exponencial entre las poblaciones censales y de las poblaciones calculadas por el Instituto Nacional de Estadística.

La tasa de mortalidad por EMN se ajustó por edad, utilizando la población estándar mundial. Para el análisis de mortalidad por cohortes se han considerado cohortes quinquenales de nacimiento, empezando por $1901 / 1905$ hasta $1941 / 1945$ y se ha excluido la mortalidad ocurrida entre los años 1951 y 1960 , debido a que los datos en este período (datos decenales) impiden la atribución de mortalidad a cohortes quinquenales. El análisis del efecto de cohortes ha sido realizado por un procedimiento gráfico en el que se representa la tasa de mortalidad por edad en ordenadas y el año de muerte en abscisas; los datos correspondientes a cada cohorte de nacimiento se unen mediante líneas. Además, mediante el paquete estadístico STATA versión 5.0 , se ha realizado un análisis de regresión de Poisson en el que la variable dependiente es el número de muertes por EMN y las variables independientes son el número de personas-año, la edad y la cohorte de nacimiento. Aunque frecuentemente se ha realizado este mismo análisis incluyendo también el año como variable independiente, se ha seguido la opinión de Moolgavkar et al. en el sentido de que este modelo (de edad-período-ohorte) no debe ser utilizado rutinariamente, porque no es enteramente satisfactorio ninguno de los métodos que corrigen el problema de la identificabilidad $^{13}$.

\section{RESULTADOS}

Tanto en hombres como en mujeres, la mortalidad por EMN ha seguido una evolución en U, descendiendo hasta $1969 \mathrm{y}$ aumentando desde entonces, aunque la mortalidad en 1992 es aún inferior a la que se produ- 
cía en los primeros años de la década de los 50 (figura 1). En mujeres la mortalidad ha sido inferior a la de los varones en todos los años y en todos los grupos de edad analiza- dos. La razón de mortalidad en varones/mortalidad en mujeres ha permanecido aproximadamente constante (mínimo: 1,18 en 1964; máximo: 2,06 en 1984, media: 1,6).

\section{Figura 1}

Mortalidad por enfermedad de la motoneurona, ajustada por edad. España, 1951-1992.

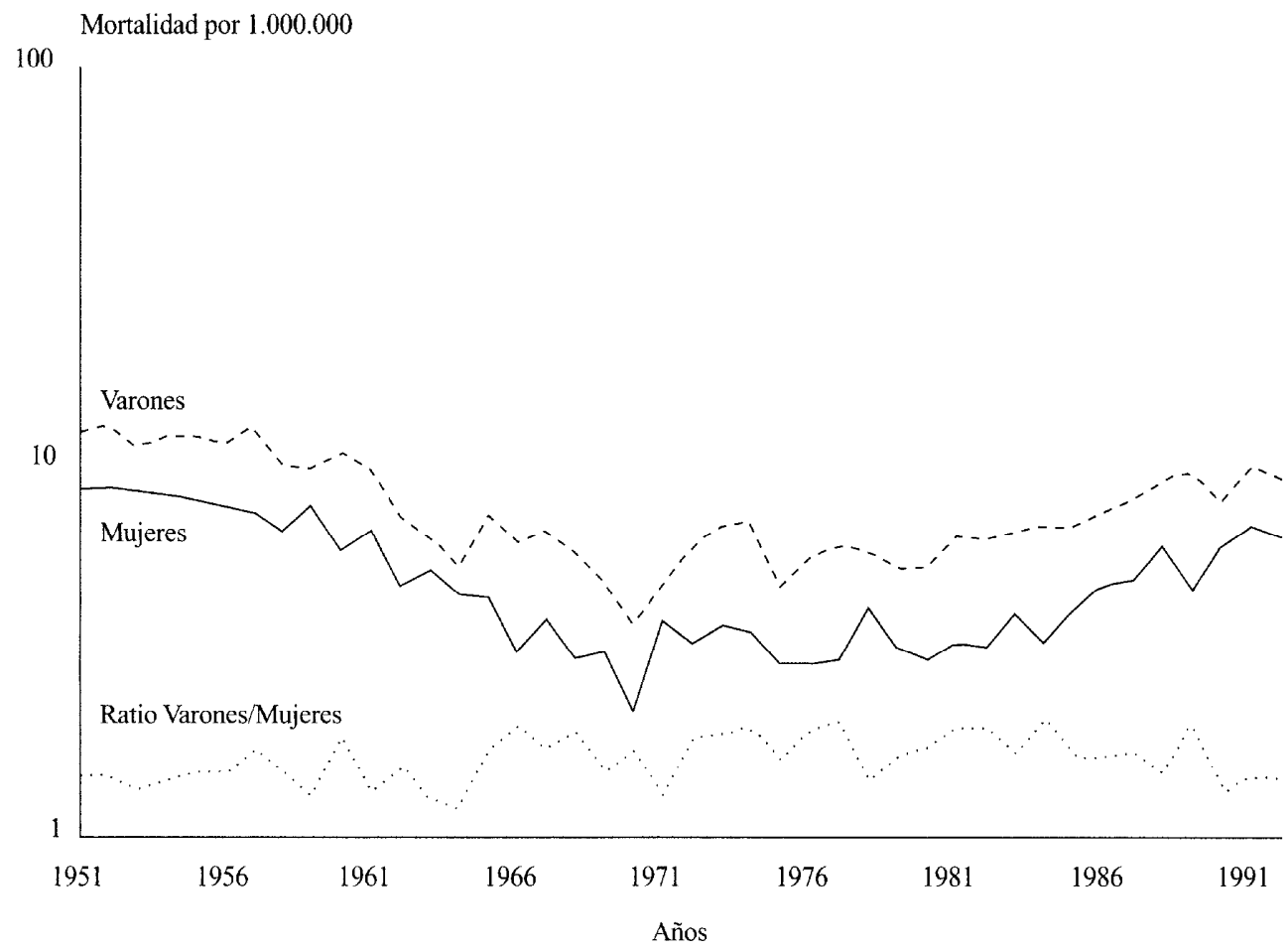

En las figuras 2 y 3 se muestra la mortalidad específica por edades. Para evitar figuras excesivamente sobrecargadas de líneas, se han seleccionado tres edades: 45-49, 60-64 y 85 y más (ésta última sólo en el periodo 1971-1992), representativas de rangos de mortalidad precoz, media y tardía, respectivamente. Pueden observarse tres tendencias diferentes: aumento en la mortalidad precoz durante todo el período (45-49 años, un patrón similar se obtiene en todo el rango 35-49), aumento desde 1970 en la mortalidad intermdia (60-64 años, patrón si- milar en el rango de edades 50-74) y descenso en la mortalidad tardía ( 85 años y más, representativo del rango 75 y más).

Para conocer si los cambios en la mortalidad siguen un patrón relacionado con la cohorte de nacimiento, se construyeron las figuras 4 (varones) y 5 (mujeres), en las que se presenta la edad de muerte en abscisas, la tasa de mortalidad en ordenadas (escala logarítmica) y cada línea representa una cohorte de nacimiento. En ambos géneros puede observarse un aumento de morta- 
Figura 2

Mortalidad por enfermedad de la motoneurona, edades seleccionadas. Varones.

Tasa de mortalidad (por 100.000)

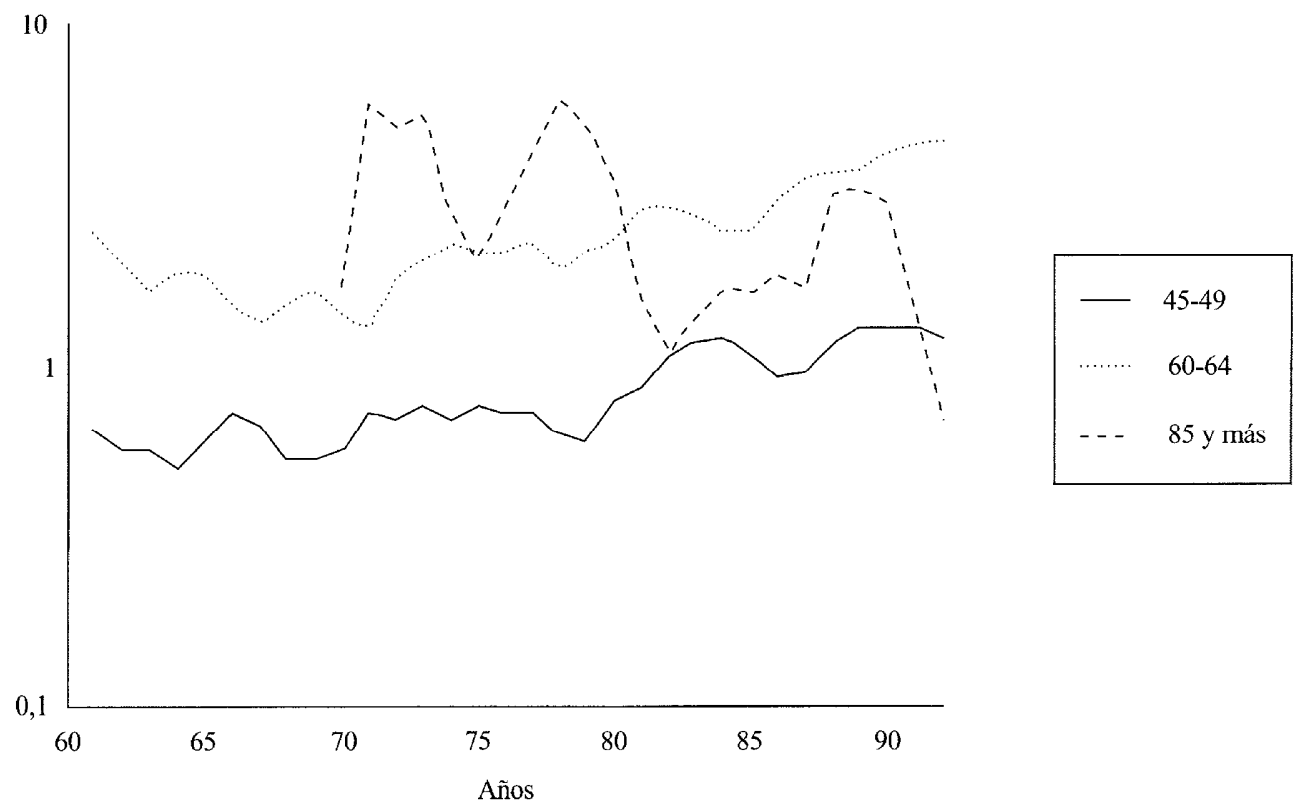

lidad cuanto más tardía es la cohorte; las dos primeras cohortes, nacidas en 19011905 y $1906-1910$, se presentan aproximadamente superpuestas, y lo mismo ocurre con las dos últimas (nacidas en 1936-1940 y 1941-1945). En cualquier cohorte la mortalidad aumenta con la edad, sin mostrar un descenso en las edades más avanzadas.

Los resultados de la regresión de Poisson se presentan en la tabla 1. Los riesgos relativos mayores que 1 en las variables edad y cohorte indican que la mortalidad por EMN aumenta con la edad y con la cohorte de nacimiento. En los hombres, el riesgo de morir por EMN aumenta un 8,5\% con cada año de edad (riesgo relativo $=1,085)$ y un $11,0 \%$ con cada cohorte (quinquenal) de nacimiento (riesgo relativo $=1,021$ para cada año). En las mujeres el riesgo aumenta un $8,5 \%$ con cada año de edad (riesgo relativo $=1,085$ ) y un $15,0 \%$ con cada cohorte quin- quenal (riesgo relativo $=1,028$ anual). En los dos modelos las asociaciones de edad y cohorte con la mortalidad son significativos para una $\mathrm{p}<0,05$.

Tabla 1

Análisis de cohortes: resultados de la regresión de Poisson (variable dependiente: número de muertes por EMN).

\begin{tabular}{|cccc|}
\hline \multirow{3}{*}{ Género } & Variable & $\begin{array}{c}\text { Riesgo } \\
\text { relativo }\end{array}$ & $\begin{array}{c}\text { Intervalo de } \\
\text { confianza al } \\
95 \%\end{array}$ \\
\hline \multirow{2}{*}{ Mujones } & Edad & 1,085 & $1,076-1,091$ \\
\cline { 2 - 4 } & Cohorte & 1,021 & $1,017-1,025$ \\
\cline { 2 - 4 } & Edad & 1,085 & $1,079-1,091$ \\
\cline { 2 - 4 } & Cohorte & 1,028 & $1,024-1,032$ \\
\hline
\end{tabular}




\section{Figura 3}

Mortalidad por enfermedad de la motoneurona, edades seleccionadas. Mujeres.

Tasa de mortalidad (por 100.000)

10

0,1

60

65

70

75

80

85

90

Años

Figura 4

Mortalidad por edades causada por la enfermedad de la motoneurona en los varones. Cada línea indica una cohorte quinquenal de nacimiento.

Tasa de mortalidad (por 100.000 )

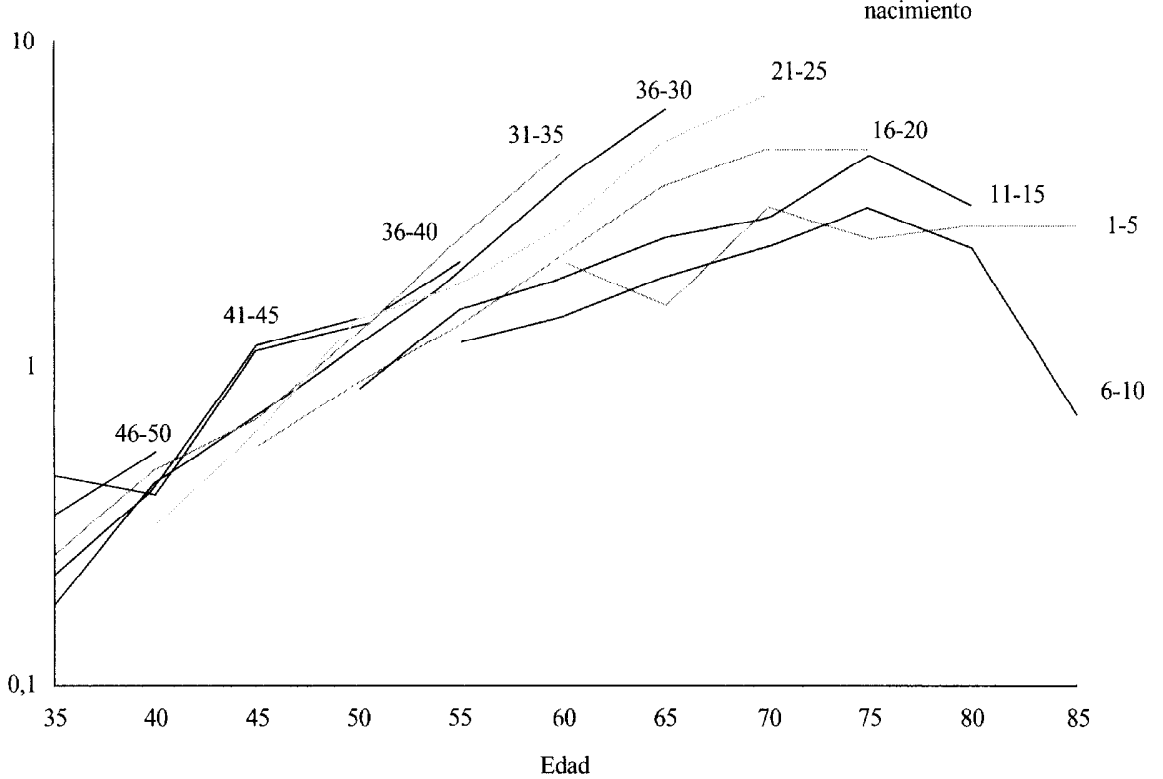


Figura 5

Mortalidad por edades causada por la enfermedad de la motoneurona en las mujeres. Cada línea indica una cohorte quinquenal de nacimiento.

Tasa de mortalidad (por 100.000)

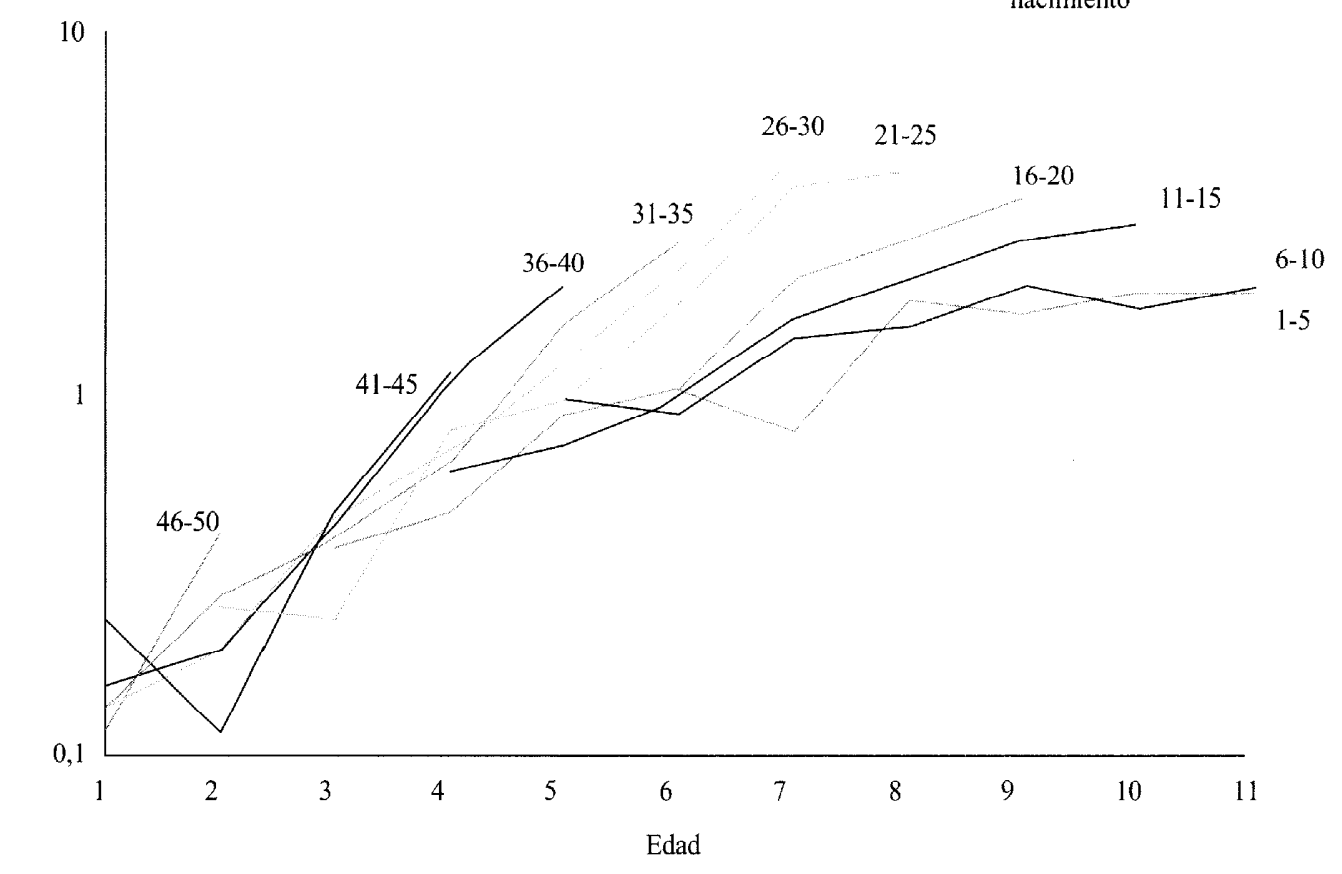

Cohorte de nacimiento

\section{DISCUSIÓN}

La mortalidad por EMN está aumentando un 2,1\% anual en varones y un 2,4\% anual en mujeres en España desde 1970, como consecuencia de un efecto de cohorte. En un aumento de la mortalidad pueden influir concurrentemente los factores que aumenten la incidencia, los que aumenten la letalidad y los cambios en la calidad del diagnóstico y de la certificación. Como limitación del estudio debe considerarse que los datos del Instituto Nacional de Estadística recogen la causa inmediata de muerte, pero no las causas intermedia y fundamental. Aunque no puede desecharse de manera absoluta, un cambio en la calidad del diagnóstico no es previsible en la EMN, puesto que se trata de una enfermedad de clínica muy llamativa, cuyo diagnóstico nomal- mente no debe pasar desapercibido; sí es posible que se hayan producido cambios como consecuencia de la aparición de nuevos métodos diagnósticos (resonancia magnética) y por la extensión de la asistencia a toda la población, así como la mayor disponibilidad de especialistas en Neurología. El estudio que conocemos sobre la validez del certificado de defunción en la EMN lo considera como una fuente de datos adecuada para su uso en epidemiología analítica ${ }^{14}$. En teoría, un aumento en la letalidad de la EMN podría estar causado por alguno de los siguientes factores: un aumento en la mortalidad por infecciones intercurrentes, un empeoramiento en los cuidados médicos y paliativos y en la ayuda social, o un cambio en la historia natural de la enfermedad. En el período analizado, los dos primeros factores han debido evolucio- 
nar precisamente en el sentido contrario, mientras que el tercer factor (un cambio en la historia natural de la ELA) es poco creíble en un período tan corto y no ha sido descrito hasta el momento (por ejemplo, no se ha informado de un descenso en la supervivencia a los 3 ó 5 años). Porlo tanto, se debe pensar que el aumento en la mortalidad por EMN se ha originado en un aumento primario de la incidencia.

Nuestros resultados sugieren que las cohortes nacidas en España desde 1901 a 1945 han estado expuestas de manera creciente a algún(os) factor(es) causal(es) de la EMN, y que este aumento en la exposición es similar en varones y en mujeres. En las últimas cohortes estudiadas parece frenarse esta tendencia al aumento. Entre los factores ambientales que se han sugerido como posibles hay que incluirla exposición a campos electromagnéticos (con un riesgo relativo aproximadamente de 2 cuando nos referimos a la exposición ocupacional) ${ }^{15-17}$, los déficits de calcio y de selenio ${ }^{18}$, los niveles altos de plomo ${ }^{19}$ y otros metales ${ }^{20}$, la exposición al radón ${ }^{21}$ y a lluvia radiactiva ${ }^{22}$, exposición ocupacional a solventes ${ }^{23}$ y a pintu$\operatorname{ras}^{24}$ y la exposición a insecticidas organo$\operatorname{clorados}^{25}$. Este trabajo, de carácter ecológico, no está diseñado para identificar los factores de riesgo que han influido en la evolución de la EMN, por lo que son necesarios otros estudios para analizar específicamente los factores de riesgo sugeridos.

Veiga-Cabo et al no encuentran - gráficamente- un aumento de la mortalidad por EMN en las edades precoces y atribuyen el incremento de cada año al grupo de edad 60-69 años, fundamentalmente ${ }^{11}$. Esta aparente contradicción con nuestros hallazgos se debe a la escala de representación gráfica. En la escala aritmética utilizada por Veiga-Cabo et al, las variaciones en la mortalidad ocurridas en los grupos de menor edad, que son los que tienen menor tasa, son imposibles de apreciar; es necesaria una presentación de los resultados en escala logarítmica para poder observar que realmente se está produciendo un incremento en estos grupos de menor edad, que es incluso más precoz que en cualquier otro.

La principal dificultad para la comparación con series internacionales es que en la mayoría de trabajos longitudinales se realizó un análisis gompertziano ${ }^{1,3,6,9}$. En estos casos la finalidad del estudio no es el análisis de cohortes sino el análisis de factores competitivos de muerte. Nuestros resultados (aumento de la mortalidad también en edades precoces) contradicen la hipótesis de que el aumento de mortaldiad por EMN en España puede deberse a un aumento en la esperanza de vida ${ }^{26}$.

En conclusión, la mortalidad por EMN en España está aumentando desde 1970, después de haber descendido en la década anterior. Cuando se analiza por cohortes, se encuentra que el riesgo de muerte por EMN ha ido aumentado desde la cohorte nacida a comienzos del siglo $\mathrm{xx}$ hasta la nacida en los años 40 .

\section{BIBLIOGRAFÍA}

1. Neilson S, Robinson I, Alperovitch A. Rising amyotrophic lateral sclerosis mortality in France 1968-1990: increased life expectancy and inter-disease competition as an explanation. J Neurol 1994; 241:448-55.

2. Durrleman $S$, Alperovitch $A$. Increasing trend of ALS in France and elsewhere: are the changes real? Neurology 1989; 39:768-73.

3. Chio A, Magnani C, Shiffer D. Gompertzian analysis of amyotrophic lateral sclerosis mortality in Italy, 1957-1987; application to birth cohorts. Neuroepidemiology 1995; 14:269-77.

4. Cio A, Magnani C, Shiffer D. Amyotrophic lateral sclerosis mortality in Italy, 1958 to 1987: a cross-sectional and cohort study. Neurology $1993 ; 43: 927-30$.

6. Neilson S, Robinson I, Nyomen EH. Longitudinal analysis of amyotrophic lateral sclerosis mortality in Norway, 1966-1989: evidence for a susceptible subpopulation. J Neural Sci 1994; 122 : $148-54$. 
7. Neilson S, Gunnarson LG, Robinson I. Rising mortality from motor neurone disease in Swseden 1961-1990: the relative role of increased population life expectancy and environmental factors. Acta Neurol Scand 1994; 90:150-9.

8. Neilson S, Robinson I, Hunter M. Longitudinal Gompertzian analysis of ALS mortality in England and Wales, 1963-1989: estimates of susceptibility in the gencral population. Mech Ageing Dev 1992; 64:201-16.

9. Eiggs JE. Longitudinal Gompertzian analysis of amyotrophic lateral sclerosis mortality in the U.S., 1977-1986: evidence for an inherently susceptible population subset. Mech Ageing Dev 1990; 55:207-20.

10. Stallones L, Kasarkis EJ, Stipanowich C, Snider G. Secular trends in mortality rates from motor neuron disease in Kentucky 1964-1984. Neuroepidemiology $1989 ; 8: 68-78$.

11. Veiga-Cabo J, Almazan-Isla J, Sendra-Gutiérrez JM, de Pedro-Cuesta J. Differential features of motor neuron disease mortality in Spain. Int $\mathbf{J}$ Epidemiol 1997; 26:1924-32.

12. López-Vega JM, Calleja J, Combarros O, Polo JM, Berciano J. Motor neuron disease in Cantabria. Acta Neurol Scand 1988; 77:1-5.

13. Moolgavkar SH, Lee $J \Lambda H$, Stevens RG. Analysis of vital statistics data. En: Rothman KJ, Greenland S. Modern Epidemiology. Lippincott-Raven, 2. ${ }^{a}$ ed., Filadelfia, 1998.

14. Chio A, Magnani C, Oddenino E, Tolardo G, Shiffer D. Accuracy of death certificate diagnosis of amyotrophic lateral sclerosis. J Epidemiol Community Health 1992; 46:517-8.

15. Johansen C, Olsen JH. Mortality from amyotrophic lateral sclerosis, other chronic disorders and electric shocks among utility workers. Am J Epidemiol 1998; 148:362-8.

16. Savitz DA, Checkoway H, Loomis DP. Magnetic field exposure and neurodegenerative disease morftality among electric utility workers. Epidemiology 1998; 9:398-404.
17. Savitz DA, Loomis DP, Tse CK. Electrical occupatiions and neurodegenerative disease: analysis of U.S. mortalith data. Arch Environ Health 1998; 53:71-4.

18. Emard JF, Thouez JP, Gauvreau D. Neurodegenerative diseases and risk factors: a literature review. Soc Sci Med 1995; 40:847-58.

19. Guidetti D, Bondavalli M, Sabadini R, Marcello N, Vinceti M, Cavalletti S, et al. Epidemiological survey of amyotrophic lateral sclerosis in the province of Reggio Emilia, Italy: influence of environmental cxposure to lead. Ncurocpidemiology $1996 ; 15: 301-12$

20. Zhang ZX, Anderon DW, Mantel N, Roman GC Motor neuron disease on Guam: geographic and familial occurrence, 1956-85. Acta Neurol Scand $1996 ; 94: 51-9$

21. Neilson S, Robinson I, Rose FC. Ecological correlates of motor neuron disease mortality: a hypothesis concerning an epidemiological association with radon gas and gamma exposure. $\mathrm{J}$ Neurol 1996; 243: 329-36.

22. Neilson S. Robinson I, Rose FC. Mortality from motor neuron disease in Japan, 1950-1990: association with radiactive fallout from atmospheric weapons testing. J Neurol Sci 1995; 134:61-6.

23. Chancellor AM, Slattery JM, Fraser H, Warlow CP. Risk factors for motor neuron disease: a case-control study based on patients from the Scottish Motor Neuron Disease Register. J. Neurol Neurosurg Psychiatry 1993; 56:1200-6.

24. Graham AJ, Macdonald AM, Hawkes CH. British motor neuron disease twin study. J Neurol Neurosurg Psychiatry 1997; 62:562-9.

25. Fonseca RG, et al. Chronic motor neuron disease possibility related to intoxication with organochlorine insecticides. Acta Neurol Scand 1993; 88:56-8.

26. Neilson S, Robinson I, de Pedro-Cuesta J, Veiga-Cabo J. Decline and rise of mortality from motor neuron disease in Spain, 1960-1989: demographic, environmental and competitive influences. Neuroepidemiology 1996;15:180-91. 